\title{
Un problema social: tiempos de espera en la consulta externa del Hospital Carlos Andrade Marín
}

A Social Issue: Waiting Times in the Outpatient Department in Carlos Andrade Marín Hospital

Um problema social: tempos de espera no ambulatório do Hospital Carlos Andrade Marín

\author{
Patricia Gómez \\ Universidad Andina Simón Bolívar, Sede Ecuador \\ pattyg_17@hotmail.com \\ Jairo Rivera \\ Universidad Andina Simón Bolivar, Sede Ecuador \\ jairo.rivera@uasb.edu.ec
}

DOI: https://doi.org/10.32719/25506641.2019.5.5

Fecha de presentación: 28 de enero de 2019 • Fecha de aceptación 24 de abril de 2019

Artículo de investigación

Licencia Creative Commons

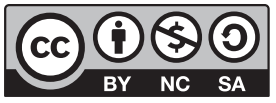




\section{Resumen}

La presente investigación analiza la gestión del tiempo de espera en la consulta externa del Hospital Carlos Andrade Marín. Inicia con una revisión de la literatura sobre la gestión de la calidad y el modelo de salud en Ecuador. Es un estudio cualitativo que se fundamenta en información primaria recopilada en entrevistas semiestructuradas a informantes clave, y en información secundaria relacionada con los procesos del hospital. Entre los principales resultados se encuentra que existen problemas internos y externos, para lo cual se desarrolla una propuesta de reducción en el tiempo de espera.

Palabras clave: sistemas de salud, atención médica, gestión de la calidad.

JEL: H75 Administración estatal, autonómica y local: salud, educación y bienestar; I11 Análisis de los mercados de asistencia sanitaria; I18 Política pública, Regulación, Sanidad pública.

\section{Abstract}

The present research analyzes the management of the waiting time in the Carlos Andrade Marín Hospital, one of the most emblematic of Quito. It begins with a literature review on quality management and the health model in Ecuador. Then, it develops the methodological section, being a qualitative study that is based on primary information collected in semi-structured interviews with key informants, and secondary information related to the Hospital's processes. Among the main results is that there are internal and external problems, for which a proposal to reduce the waiting time is developed.

Keywords: health systems, medical care, quality management.

JEL: H75 State and Local Government: Health; Education; Welfare; Public Pensions; I11 Analysis of Health Care Markets; I18 Government Policy; Regulation; Public Health.

\section{Resumo}

A presente investigação analisa o gerenciamento do tempo de espera no ambulatório do hospital Carlos Andrade Marín, um dos mais emblemáticos de Quito. Começa com uma revisão da literatura sobre gestão da qualidade e o modelo de saúde no Equador. Então passou a ser a seção metodológica, sendo um estudo qualitativo baseado em informações primárias coletadas em entrevistas semiestruturadas com informantes-chave e informações secundárias relacionadas aos processos do Hospital. Entre os principais resultados está a existência de problemas internos e externos, para os quais é apresentada uma proposta de redução do tempo de espera.

Palavras-chave: sistemas de saúde, assistência médica, gestão da qualidade.

JEL: H75 Administração estadual, autônoma e local: saúde, educação e bem-estar; I11 Análise de mercados de saúde; I18 Políticas públicas, regulação, saúde pública. 


\section{Introducción}

" $\cap$ oda persona tiene derecho a un nivel de vida adecuado que le asegure, así como a su familia, la salud y el bienestar" (ONU 1948, 7). En

Ecuador, los principales derechos de los ciudadanos se resumen en el artículo 3 de la Constitución de la República (EC 2008), cuya primera letra, establece como deber primordial que el Estado debe garantizar para todos los habitantes del país, sin discriminación alguna: "el acceso a la educación, salud, alimentación, seguridad social y el agua". En ese mismo contexto, la Ley Orgánica de Salud (EC 2012), en su artículo 1, hace énfasis a la garantía de la salud como derecho de los ecuatorianos, determinando que es importante "regular las acciones que permitan efectivizar el derecho universal a la salud consagrado en la Constitución Política de la República".

Por tal razón, a través del tiempo se han priorizado aquellas políticas públicas enfocadas principalmente en la salud, las cuales pretenden resolver los problemas del país de manera holística, que afectan directamente la calidad de vida de sus habitantes. Sin embargo, aún no ha sido tarea fácil resolver los temas relacionados con la garantía de la atención médica oportuna en las unidades públicas del país, donde los pacientes en algunas ocasiones deben esperar semanas o meses para ser atendidos por el personal médico.

El presente estudio tiene como objetivo principal determinar acciones, mediante la gestión por procesos, que permitan reducir el tiempo de espera de los pacientes -desde que surge la necesidad de la cita médica hasta que es efectivamente atendido por un especialista en la consulta externa- en el Hospital Carlos Andrade Marín (HCAM). El HCAM es uno de los hospitales más emblemáticos del Instituto Ecuatoriano de Seguridad Social, y es una de las tres unidades correspondientes al tercer nivel de atención, atiende patologías de alta complejidad, cuya organización ha sido foco de interés para la población y es precisamente donde se han suscitado varias quejas por parte 
de sus usuarios. En este proceso se analiza la lista de espera del servicio de consulta externa, el proceso de asignación de turnos y se establecen estrategias mediante el uso de herramientas de calidad que permitan reducir el tiempo de espera de los pacientes para ser atendidos.

Este estudio parte de una revisión de la literatura relacionada con la gestión de procesos y la gestión de la calidad de la salud. En cuanto a la metodología, es de tipo descriptivo transversal, debido a que se ha investigado la situación en un punto del tiempo con un grupo específico de personas, utilizando el método deductivo. La recopilación de la información ha sido realizada a través de fuentes primarias, tales como personal responsable del proceso de agendamiento de citas médicas, pacientes, profesionales de la salud y funcionarios administrativos del HCAM e IESS, a quienes se les ha aplicado entrevistas individuales y grupales; y las fuentes secundarias son: las agendas de pacientes, encuesta de satisfacción, procesos documentados y documentos oficiales.

Entre los principales resultados de la investigación, se ha encontrado que existen problemas dentro de los procesos ligados a la consulta externa del Hospital Carlos Andrade Marín. Ante ello, se propusieron estrategias, actividades, riesgos y sus contramedidas, con la finalidad de resolver los problemas en el proceso de asignación de turnos; es decir, medidas que impacten en mejorar el tiempo de espera para recibir una atención efectiva por un especialista. Sin embargo, varias acciones sugeridas involucran a instancias externas al HCAM, tales como unidades médicas de la seguridad social, Ministerio de Salud Pública, Instituto Ecuatoriano de Seguridad Social, entre otras, de manera que, si no existe una cohesión entre las entidades responsables, no se podrá poner fin al problema de los tiempos de espera.

La investigación se estructura así: en la siguiente sección se realiza la revisión de la literatura sobre la gestión de la calidad y el modelo de salud en Ecuador; posteriormente, se presentan la metodología y las fuentes de información; luego, se desarrollan los resultados y su interpretación; por último, en la última sección, se presentan las conclusiones del estudio. 


\section{Revisión de literatura}

La gestión de la calidad es crucial para el mejoramiento continuo del servicio y la satisfacción del cliente. El Comité Europeo de Normalización (2015) afirma que la gestión basada en procesos tiene como objetivo principal la mejora sistemática, priorizando aquellos que son considerados como esenciales para la supervivencia de la organización; además, es importante considerar que los procesos que se encuentran interrelacionados óptimamente hacen que contribuyan de mejor manera a la eficacia y eficiencia de la institución en el logro de sus objetivos.

La estructura de los procesos está compuesta por elementos técnicos y humanos en que la solución de los mecanismos técnicos generalmente involucra trabajar sobre varias causas debido a que, normalmente, la problemática es la consecuencia de un conjunto de errores; en la identificación de los problemas involucrados en la calidad, es importante el análisis de un conjunto de mecanismos como mano de obra, materia prima, maquinaria, métodos, medioambiente, medición, mejora, gestión y recursos (Blandón 2013).

Los centros hospitalarios alrededor del mundo funcionan a partir de una cantidad enorme de procesos que se encuentran interrelacionados entre distintos departamentos; en este ciclo intervienen insumos, mecanismos, controles y salidas; a su vez, importa el alcance, los actores y sus actividades, informes documentados, evaluaciones y la mejora continua (Pérez 2004); en ese sentido, resulta de crucial importancia la gestión de la calidad de los centros hospitalarios.

En Ecuador, el Sistema Nacional de Salud se ha caracterizado por estar "fragmentado, centralizado y desarticulado en la provisión de servicios de salud" (Ministerio de Salud Pública 2012, 23). En apego a sus responsabilidades, el Ministerio de Salud Pública oficializa el Modelo de Atención Integral de Salud (MAIS) que plasma las directrices específicas que han permitido operativizar la normativa legal, especialmente la estipulada en la Constitución de la República del Ecuador; la gestión se realiza mediante la aplicación de un modelo de salud y la articulación de la Red Pública Integral de Salud.

Este modelo tiene como objetivo incorporar una estrategia que integre a cuatro niveles de atención médica, las cuales se clasifican según su capacidad 
resolutiva, permitiendo así que los servicios se orienten hacia la promoción de salud y prevención de enfermedades. Así, también, prevé fortalecer el proceso de recuperación, rehabilitación y cuidados paliativos, garantizando la calidad de la atención, mediante el acceso efectivo, universal y gratuito.

El Manual MAIS del Ministerio de Salud Pública (2012) sostiene que el primer nivel de atención es la puerta de entrada a la red y se compone de atención extramural e intramural. El segundo nivel de atención representa el escalón inmediato para la referencia de aquellos casos que no han sido resueltos en el primer nivel, y está constituido por aquellos establecimientos que brindan servicios de atención ambulatoria especializada, cirugías, hospitalización y hospitalización del día, y presenta dos categorías, ambulatoria (servicios sin internación) y hospitalización (servicios con internación). El tercer nivel está compuesto por establecimientos de referencia nacional, donde se brinda atención a pacientes con patologías más complejas, de todos los lugares del país, mediante el uso de equipos con tecnología de punta y se encuentran preparados para realizar trasplantes. El cuarto nivel brinda servicios especializados, cuyo objetivo principal se centra en realizar investigaciones en materia de salud con base a experimentos clínicos.

La estructura desarrollada desde el MAIS propone una organización del sistema de salud, sin embargo, su implementación todavía presenta desafíos, pues la existencia de "largos tiempos de espera es un problema común en hospitales y centros de salud urbanos en el Ecuador" (Hermida, Laspina e Idrovo 1996, 95).

Para el Ecuador existen investigaciones interesantes relacionadas con los tiempos de espera en centros hospitalarios, aunque todavía resultan escasos. Dichas investigaciones, por un lado, se enfocan en el análisis de los procesos relacionados con la gestión de la calidad en los tiempos de espera; y, por otra parte, se asocian con análisis estadísticos de optimización de funciones objetivos relacionadas con la minimización de los tiempos de espera.

La investigación realizada por Hermida, Laspina e Idrovo (1996) pertenece al primer grupo de investigaciones e indaga sobre los tiempos de espera en un centro de salud del Ecuador, ubicado en La Troncal (Cañar); en este estudio se aborda el problema mediante técnicas relacionadas con el mejoramiento de la calidad, a través del análisis de procesos. Como resultado se encontró que existen procesos críticos relacionados con la documentación de 
las personas, asignación de turnos a los médicos, horarios de saturación, y poca interacción entre los distintos actores.

El estudio realizado por Gutiérrez, Recalde, Guevara y Rivadeneira (2009) se asocia al segundo tipo de investigaciones, y desarrolla una propuesta de optimización de los tiempos de espera del Hospital Eugenio Espejo ubicado en Quito; para ello, mediante procesos de optimización matemática, se realizó una modelización, simulación y minimización de los tiempos de espera; y se obtuvo como resultado que existen problemas relacionados con un número inadecuado de personal, horarios de atención, y agendamiento de citas. En esa misma línea, Velázquez y Vinueza (2017) realizan un análisis de teoría de colas a los sistemas de gestión de los centros de atención primaria, en los que, optimizando la distribución de pacientes y médicos y evitando cuellos de botella, se pueden reducir los tiempos de espera de las personas.

Del análisis de la literatura se encuentra que los tiempos de espera son importantes dentro de la calidad de los centros de atención de salud; existen investigaciones relacionadas con la temática, pero aplicadas en otros contextos, en distinto tiempo y con diversas metodologías. En ese sentido, este estudio pretende ser un aporte al análisis de los tiempos de espera del HCAM, aplicando técnicas relacionadas con la gestión de la calidad.

El HCAM es uno de los hospitales más emblemáticos del Instituto Ecuatoriano de Seguridad Social dentro de la provincia de Pichincha. Esta institución ha brindado atención a sus pacientes durante 47 años, el hospital es de tercer nivel y cuenta con servicios de atención ambulatoria, hospitalización (en áreas clínicas y quirúrgicas), además de los servicios de ambulancia, cuidados intensivos y emergencia (tanto de adultos como de pediátricos); $\mathrm{y}$, durante los últimos años se han realizado trasplantes de hígado, riñón, córnea y pulmón.

\section{Metodología}

Esta investigación usa una metodología cualitativa que se fundamenta en información primaria y secundaria. Por una parte, las fuentes primarias de información han sido las entrevistas en profundidad y grupos focales a funcionarios y pacientes. Por otra parte, para el análisis de procesos e iden- 
tificación del estado situacional, se han utilizado fuentes secundarias, tales como bases de datos, archivos públicos de la organización, y normativa legal nacional; adicionalmente, se ha realizado una revisión literaria de documentos oficiales y revistas científicas que se establecen mecanismos para recopilar información, realizar un diagnóstico, y proponer soluciones para resolver el problema.

En este estudio se han utilizado herramientas de calidad que permitan organizar al proceso de asignación de turnos en la consulta externa y reducir el tiempo de espera del paciente para recibir una atención efectiva. En contexto con lo anterior, es importante citar al Comité Europeo de Normalización (2015), el cual indica que, mediante procesos, se puede mejorar la satisfacción del cliente, satisfaciendo sus necesidades, contribuyendo a la eficacia y eficiencia de la organización para lograr sus objetivos.

Finalmente, se ha sistematizado y consolidado la información recopilada, con el fin de determinar las causas y efectos del problema, proponer el plan de acción y, a su vez, evaluar riesgos y sus contramedidas.

\section{Levantamiento de información}

Para el diagnóstico del proceso de asignación de citas médicas en la consulta externa, se han realizado entrevistas individuales y grupales. El equipo ha sido conformado por 9 pacientes de la consulta externa, personal de atención al cliente involucrado en el proceso, y funcionarios médicos y administrativos tomadores de decisiones. Se ha realizado una selección aleatoria de pacientes, atendidos por primera vez en una especialidad, durante la salida o ingreso de su consulta. El levantamiento de información se fundamenta en el muestreo teórico, y ha sido generado hasta que sus respuestas se volvieron recurrentes.

A partir de los datos proporcionados, se han agrupado ideas afines, como línea base para identificar causas, efectos, y estrategias orientadas a resolver problemas, determinar los posibles riesgos y sus contramedidas, considerando la posibilidad de implementación. 


\section{Herramientas de planificación y gestión de la calidad}

Con la finalidad de determinar alternativas para la resolución de problemas, un diagnóstico e identificar estrategias, a continuación, se detallan los métodos desarrollados en este estudio (Camisón, Cruz y Gonzales 2006):

Tabla 1

Aplicación de herramientas de calidad para la resolución de problemas

\begin{tabular}{|l|l|l|}
\hline \multicolumn{1}{|c|}{ Herramientas } & \multicolumn{1}{|c|}{ Identificación del problema } & \multicolumn{1}{c|}{ Definir el problema } \\
\hline ¿Cuál es el problema? & Identificar problemas. & Diagrama de afinidad. \\
\hline ¿Cuáles son las causas? & $\begin{array}{l}\text { Identificar causas raíz del } \\
\text { problema. }\end{array}$ & Diagrama de relaciones. \\
\hline ¿De qué forma se lo resuelve? & $\begin{array}{l}\text { Identificar todas las soluciones } \\
\text { del problema. }\end{array}$ & Diagrama de árbol. \\
\hline ¿Qué decisión tomar? & Seleccionar la mejor solución. & $\begin{array}{l}\text { Diagrama de priorización o } \\
\text { Diagrama matricial. }\end{array}$ \\
\hline ¿Cuándo y cómo actuar? & $\begin{array}{l}\text { Planificar la puesta en práctica } \\
\text { de la mejor solución. }\end{array}$ & $\begin{array}{l}\text { Diagrama de proceso de } \\
\text { decisión o } \\
\text { Diagrama de flechas. }\end{array}$ \\
\hline
\end{tabular}

Fuente: Vilar, Gómez y Tejero (1997).

Elaboración propia.

Con el diagrama de afinidad se han consolidado las ideas afines, agrupándolas -entre sí- en factores más amplios. Con base en estos últimos se ha construido el diagrama de relaciones, el cual sirve para identificar causas (mayor número de flechas salientes) y efectos (mayor número de flechas entrantes), determinándose así aquellos factores clave. A partir de la detección de las causales, con el diagrama de árbol, se ha organizado y graficado estrategias con sus respectivas actividades, para cumplir el objetivo de esta investigación. El diagrama de priorización, para la toma de decisiones, jerarquiza actividades, temas, y características de productos o servicios, a partir de un criterio de asignación de puntajes y de su ponderación. Finalmente, el diagrama de decisiones, permite determinar riesgos y medidas de contingencia que se presenten en el plan de acción. 


\section{Resultados}

\section{Determinación de problemas identificados en el proceso de agendamiento}

En el año 2017, el 15\% de las citas atendidas provienen de unidades médicas de primer nivel, ocupándose turnos que pueden ser asignados a otros pacientes de mayor prioridad. También se ha identificado que existen pacientes que, por su ubicación geográfica, deben ser atendidos en otras unidades médicas (provenientes de Guayas, Santa Elena, El Oro, Loja, entre otras provincias).

Seguidamente, se ha realizado una revisión del proceso de agendamiento de citas médicas, $\mathrm{y}$, mediante la documentación y la experiencia de los funcionarios, se han levantado observaciones identificando causas y efectos del problema. Este análisis tiene tres componentes (internos, externos y pacientes).

\section{Problemas internos para el agendamiento de citas}

- Ausencia de definiciones internas: existen casos que carecen de términos homologados dentro de la organización o definidos de manera oficial, afectando al entendimiento de las metodologías, y procesos internos.

- Falta de aplicativos informáticos estandarizados: cuando la unidad médica no dispone de la capacidad para asignar un turno, el usuario ha realizado una visita innecesaria, y su necesidad no es registrada en algún aplicativo informático; por tal razón la cita no es asignada automáticamente cuando existen espacios disponibles.

- Falta de revisión constante de la normativa: el uso de términos que se maneja en el proceso no corresponde a los descritos en la normativa y/o manuales vigentes del MSP.

- Los médicos se ausentan y los pacientes tienen que volver al final de la lista: existen casos en que el médico genera permisos, vacaciones, congresos, reuniones, entre otros, lo que ocasiona el reagendamiento para el 
final de la lista de espera, dilatando el tiempo para recibir una atención efectiva.

- Falta de contrarreferencia de los pacientes por parte de los médicos del HCAM: existe dificultad para que el médico envíe al paciente a una unidad inferior, porque este prefiere atenderlo y no dejar su caso para poder monitorearlo, o porque no existe disponibilidad.

- Falta de un filtro médico adecuado en el triaje $e^{1}$ de agendamiento: una vez que se le asigna un turno médico, existe el triaje que se determina si el paciente es de tercer nivel, adicionalmente se revisa la documentación para ser atendido. Sin embargo, el personal asignado para este filtro no es médico sino administrativo, por tal razón el criterio no es adecuado.

- No se dispone de alternativas de solución para la gestión del requerimiento del usuario al momento que lo solicita: cuando el paciente debe ser atendido en otra unidad médica de la red de salud, no se garantiza su turno y más aún la resolución de su problema de salud.

\section{Problemas externos para el agendamiento de citas}

- Ausencia de un proceso sistematizado para las unidades médicas del III nivel: el proceso de agendamiento de citas médicas no es divulgado y homologado con el resto de unidades médicas, lo cual impide que exista una cohesión que permita generar colaboración entre sí.

- Falta de control y evaluación del proceso para la mejora continua: no se dispone de un mecanismo de monitoreo, seguimiento y evaluación del proceso, lo que no permite identificar debilidades para realizar mejoras.

- Agendas médicas abiertas para todos los niveles de atención: el hospital tiene abiertas las agendas médicas. Esto implica que cualquier casa de salud (pública o privada), de los diferentes niveles de atención, pueda asignar una cita médica.

- Las unidades del nivel de inferior tienen bloqueadas las agendas para asignación de turno: el cierre de las agendas de los hospitales del segundo nivel ha hecho que el hospital no pueda asignar turnos. Y, al no encon-

1. Se entiende como la categorización del paciente, según su prioridad de atención. Entrevista. 
trar alternativas para evacuar al paciente, debe seguir atendiéndolo. Las justificaciones se alinean para un colapso en los filtros de atención (nivel I y II).

- No existe en la ciudad de Quito puertas de entrada a la red para la atención en salud: al no existir en el área urbana de Quito unidades del primer nivel, las del segundo colapsan al funcionar como puerta de entrada para resolver los problemas de salud de la población.

\section{Análisis de la problemática mediante entrevistas con los pacientes}

- El usuario reacciona de manera negativa cuando no se le asigna un turno: el paciente ha indicado que tardan de dos a seis meses para recibir una atención efectiva por un especialista. En el caso de los dos meses o menos, se debe a la gestión de los médicos para asignarles citas. Generalmente los pacientes se ven la necesidad de soportar la espera por la falta de alternativas y de ingresos para seguir un tratamiento en un hospital privado.

- El paciente no está siendo correctamente informado sobre el funcionamiento de la red pública de salud: los pacientes, por desconocimiento, acuden a la unidad médica exigiendo atención, sin haber sido valorados previamente en un nivel inferior. Sin embargo, manifiestan su inconformidad al no acceder a un turno. Así también los usuarios se niegan a recibir ayuda de otra unidad médica, exigiendo ser atendidos en el HCAM.

- Los diagnósticos médicos no son acertados oportunamente: algunos usuarios han coincidido en que solicitaron cita médica en los niveles inferiores, consiguiéndola después de un largo tiempo; sin embargo, con el tratamiento no hubo mejoría en su salud, y luego de un tiempo han acudido a la emergencia del HCAM; en ese momento se le indica la existencia de enfermedades irreversibles por falta de oportunidad en la atención. 


\section{Análisis de resolución de problemas mediante el uso de herramientas de calidad}

\section{Diagrama de afinidad}

Una vez recopilada la información de todos los actores involucrados, se han agrupado ideas relacionadas, en componentes más amplios, según la siguiente tabla:

Tabla 2

Diagrama de afinidad

\begin{tabular}{|c|c|}
\hline Lluvia de ideas (problemas) & Agrupación o afinidad \\
\hline Mal uso de los términos involucrados en el proceso. & \multirow{2}{*}{$\begin{array}{l}\text { Falta de revisión constante de } \\
\text { la normativa. }\end{array}$} \\
\hline Falta de revisión de la normativa. & \\
\hline $\begin{array}{l}\text { Falta de definiciones claras para determinar un estándar respecto } \\
\text { del tiempo que el paciente debe esperar para ser atendido sin que } \\
\text { afecte su condición. }\end{array}$ & $\begin{array}{l}\text { Ausencia de definiciones inter- } \\
\text { nas. }\end{array}$ \\
\hline Cierre de agendas del segundo nivel de atención. & \multirow{2}{*}{$\begin{array}{l}\text { Las unidades del nivel de in- } \\
\text { ferior tienen bloqueadas las } \\
\text { agendas para asignación de } \\
\text { turno. }\end{array}$} \\
\hline $\begin{array}{l}\text { Las unidades de segundo nivel no siempre reciben pacientes que } \\
\text { deben ser enviados desde el HCAM. }\end{array}$ & \\
\hline $\begin{array}{l}\text { El primer nivel y prestadores externos pueden agendar citas al } \\
\text { HCAM. }\end{array}$ & \multirow[t]{2}{*}{$\begin{array}{l}\text { Agendas médicas abiertas para } \\
\text { todos los niveles de atención. }\end{array}$} \\
\hline Agendas del tercer nivel abiertas para cualquier nivel de atención. & \\
\hline $\begin{array}{l}\text { Los médicos no quieren enviar a otra unidad médica a los pacien- } \\
\text { tes que no pertenecen al HCAM. }\end{array}$ & $\begin{array}{l}\text { Falta de contrarreferencia de } \\
\text { los pacientes por parte de los } \\
\text { médicos del HCAM. }\end{array}$ \\
\hline $\begin{array}{l}\text { Es cultural, los pacientes no aceptan ser atendidos en otras uni- } \\
\text { dades médicas. }\end{array}$ & \multirow{3}{*}{$\begin{array}{l}\text { El paciente no está siendo co- } \\
\text { rrectamente informado sobre el } \\
\text { funcionamiento de la red públi- } \\
\text { ca de salud. }\end{array}$} \\
\hline $\begin{array}{l}\text { Los pacientes no saben a dónde acudir para ser atendidos por un } \\
\text { especialista. }\end{array}$ & \\
\hline $\begin{array}{l}\text { Los usuarios desconocen la articulación de la red por eso exigen } \\
\text { atención en el HCAM. }\end{array}$ & \\
\hline
\end{tabular}




\begin{tabular}{|c|c|}
\hline Lluvia de ideas (problemas) & Agrupación o afinidad \\
\hline El paciente se enoja cuando no se le asigna un turno médico. & $\begin{array}{l}\text { El usuario reacciona de ma- } \\
\text { nera negativa cuando no se le } \\
\text { asigna un turno. }\end{array}$ \\
\hline $\begin{array}{l}\text { No existe un proceso sistematizado para las unidades médicas } \\
\text { del III nivel. }\end{array}$ & $\begin{array}{l}\text { Ausencia de un proceso sis- } \\
\text { tematizado para las unidades } \\
\text { médicas del III nivel. }\end{array}$ \\
\hline $\begin{array}{l}\text { No existe registro de los pacientes que no han podido ser asigna- } \\
\text { dos con una cita. }\end{array}$ & \multirow[t]{2}{*}{$\begin{array}{l}\text { Falta de aplicativos informáti- } \\
\text { cos estandarizados. }\end{array}$} \\
\hline $\begin{array}{l}\text { Falta de aplicativos informáticos para la asignación óptima de tur- } \\
\text { nos. }\end{array}$ & \\
\hline Falta de estandarización de la información. & \\
\hline No existe el seguimiento para la articulación de la red de salud. & \multirow{3}{*}{$\begin{array}{l}\text { Falta de control y evaluación } \\
\text { del proceso para la mejora con- } \\
\text { tinua. }\end{array}$} \\
\hline $\begin{array}{l}\text { Las autoridades desconocen de los inconvenientes que se presen- } \\
\text { tan en el proceso. }\end{array}$ & \\
\hline $\begin{array}{l}\text { No existe el seguimiento del proceso de agendamiento de citas } \\
\text { médicas para mejorarlo. }\end{array}$ & \\
\hline Triaje inadecuado para el agendamiento. & $\begin{array}{l}\text { Falta de criterios para realizar } \\
\text { un agendamiento adecuado. }\end{array}$ \\
\hline $\begin{array}{l}\text { El paciente en la ventanilla se entera de que no está asegurado y } \\
\text { hace una visita en vano. }\end{array}$ & \multirow{2}{*}{$\begin{array}{l}\text { No se dispone de alternativas } \\
\text { de solución para la gestión del } \\
\text { requerimiento del usuario al } \\
\text { momento que lo solicita. }\end{array}$} \\
\hline $\begin{array}{l}\text { El paciente debe acudir más de una vez para recibir una cita mé- } \\
\text { dica. }\end{array}$ & \\
\hline No existen unidades médicas de primer nivel en la ciudad de Quito. & $\begin{array}{l}\text { No existe en la ciudad de Quito } \\
\text { puertas de entrada (nivel I) a la } \\
\text { red para la atención en salud. }\end{array}$ \\
\hline $\begin{array}{l}\text { Cuando los médicos se ausentan, los pacientes tienen que volver } \\
\text { al final de la lista de espera. }\end{array}$ & $\begin{array}{l}\text { Los médicos se ausentan y los } \\
\text { pacientes tienen que volver al } \\
\text { final de la lista. }\end{array}$ \\
\hline $\begin{array}{l}\text { Los diagnósticos médicos por parte de las unidades inferiores no } \\
\text { son acertados ni oportuna. }\end{array}$ & $\begin{array}{l}\text { Los diagnósticos médicos no } \\
\text { son acertados ni oportunamen- } \\
\text { te. }\end{array}$ \\
\hline
\end{tabular}

Fuente: estudio de campo.

Elaboración propia. 


\section{Diagrama de relaciones}

A partir de las relaciones encontradas se han identificado las causas principales en la asignación de citas para la consulta externa como se muestra a continuación:

- Ausencia de definiciones internas.

- Ausencia de un proceso sistematizado para las unidades médicas del III nivel.

- Falta de control y evaluación del proceso para la mejora continua.

- No existen en la ciudad de Quito puertas de entrada (nivel I) para la red para de atención en salud.

- Los diagnósticos médicos no son acertados oportunamente.

- Falta de revisión constante de la normativa por parte de los funcionarios. El resto de factores analizados ha sido identificado como efectos (ver gráfico 1).

\section{Diagrama de árbol}

Considerando las causas identificadas previamente, a continuación, se proponen alternativas de solución, aunque no siempre puedan ser fáciles de implementarlas, debido a que dependen de factores internos y externos, pero son de alto interés a la población en general, debido al alcance hacia la red de salud del Instituto Ecuatoriano de Seguridad Social (IESS) (ver gráfico 2).

- Implementación de definiciones internas.

Las definiciones deben ser homologadas para las unidades médicas del IESS. De manera que sean determinadas por funcionarios con vasto conocimiento. Es necesario que se establezca un proceso para determinar definiciones oficiales, formalizándose en todas las unidades médicas.

- Generación de procesos sistematizados para la gestión de citas médicas, en las unidades del III nivel: es importante que la implementación, divulgación y seguimiento de procesos permitan el correcto funcionamiento del agendamiento de citas, y se recomienda que estos sean homologados para todos los hospitales. 


\section{Gráfico 1 \\ Diagrama de relaciones}

A continuación, se detallan las relaciones existentes entre cada uno de los problemas encontrados (los cuales, para su mejor manejo, han sido codificados con la inicial "P" y un numeral), con la finalidad de determinar si son causas $o$ efectos.

\begin{tabular}{|l|l|c|c|}
\hline \multicolumn{1}{|c|}{ Problemas } & P1 & P2 \\
\hline P1 & Falta de revisión continua de la normativa por parte de los funcionarios. & 0 \\
\hline P2 & Ausencia de definiciones internas. & 0 & \\
\hline P3 & $\begin{array}{l}\text { Las unidades del nivel de inferior tienen bloqueadas las agendas para asignación de } \\
\text { turno. }\end{array}$ & 0 & 0 \\
\hline P4 & Agendas médicas abiertas para todos los niveles de atención. & 0 & 0 \\
\hline P5 & Falta de contrarreferencia de los pacientes por parte de los médicos del HCAM. & 0 & 0 \\
\hline P6 & $\begin{array}{l}\text { El paciente no está siendo correctamente informado sobre el funcionamiento de la red } \\
\text { pública de salud. }\end{array}$ & 0 & 0 \\
\hline P7 & El usuario reacciona de manera negativa cuando no se le asigna un turno. & 0 & 0 \\
\hline P8 & Ausencia de un proceso sistematizado para las unidades médicas del III nivel. & 0 & 0 \\
\hline P9 & Falta de aplicativos informáticos adecuados. & 0 & 0 \\
\hline P10 & Falta de control y evaluación del proceso para la mejora continua. & 1 & 0 \\
\hline P11 & Falta de un criterio para realizar un agendamiento adecuado. & 0 & 0 \\
\hline P12 & $\begin{array}{l}\text { No se dispone de alternativas de solución para la gestión del requerimiento del usuario } \\
\text { al momento que lo solicita. }\end{array}$ & 0 & 0 \\
\hline P13 & $\begin{array}{l}\text { No existe en la ciudad de Quito puertas de entrada (nivel 1) a la red para la atención en } \\
\text { salud. }\end{array}$ & 0 & 0 \\
\hline P14 & Los médicos se ausentan, y los pacientes tienen que volver al final de la lista. & 0 & 0 \\
\hline P15 & Los diagnósticos médicos no son acertados oportunamente. & 0 & 0 \\
\hline
\end{tabular}

Fuente: estudio de campo.

Elaboración propia.

- Fortalecimiento de las puertas de entrada (I Nivel) a la red de salud: la propuesta se basa en la coordinación con el MSP, de manera que el call center del IESS pueda agendar directamente a los usuarios de primer nivel, y el MSP sea puerta de entrada. Adicionalmente, con base en los resultados obtenidos a partir de un perfil epidemiológico, se debe rees- 


\begin{tabular}{|c|c|c|c|c|c|c|c|c|c|c|c|c|c|c|}
\hline P3 & P4 & P5 & P6 & P7 & P8 & P9 & P10 & P11 & P12 & P13 & P14 & P15 & $\begin{array}{c}\text { Salidas } \\
\text { (causas) }\end{array}$ & $\begin{array}{c}\text { Entradas } \\
\text { (efectos) }\end{array}$ \\
\hline 0 & 1 & 1 & 1 & 0 & 0 & 1 & 0 & 1 & 0 & 0 & 0 & 0 & 5 & 1 \\
\hline 0 & 1 & 1 & 1 & 1 & 0 & 1 & 1 & 1 & 1 & 0 & 1 & 0 & 9 & 0 \\
\hline & 0 & 1 & 0 & 1 & 0 & 0 & 0 & 0 & 1 & 0 & 0 & 0 & 3 & 4 \\
\hline 0 & & 0 & 1 & 1 & 0 & 0 & 0 & 1 & & 0 & 0 & 0 & 3 & 6 \\
\hline 0 & 0 & & 0 & 1 & 0 & 0 & 0 & 0 & 1 & 0 & 0 & 0 & 2 & 7 \\
\hline 0 & 0 & 0 & & 1 & 0 & 0 & 0 & 0 & 0 & 0 & 0 & 0 & 1 & 11 \\
\hline 0 & 0 & 0 & 0 & & 0 & 0 & 0 & 0 & 0 & 0 & 0 & 0 & 0 & 12 \\
\hline 1 & 1 & 0 & 1 & 1 & & 1 & 1 & 0 & 1 & 0 & 1 & 0 & 8 & 0 \\
\hline 1 & 1 & 0 & 1 & 0 & 0 & & 0 & 0 & 0 & 0 & 0 & 0 & 3 & 4 \\
\hline 1 & 1 & 1 & 1 & 1 & 0 & 1 & & 1 & 1 & 0 & 1 & 0 & 10 & 2 \\
\hline 0 & 0 & 1 & 1 & 1 & 0 & 0 & 0 & & 1 & 0 & 0 & 0 & 4 & 5 \\
\hline 0 & 0 & 0 & 1 & 1 & 0 & 0 & 0 & 0 & & 0 & 0 & 0 & 2 & 8 \\
\hline 1 & 1 & 1 & 1 & 1 & 0 & 0 & 0 & 1 & 1 & & 0 & 0 & 7 & 0 \\
\hline 0 & 0 & 0 & 1 & 1 & 0 & 0 & 0 & 0 & 0 & 0 & & 0 & 2 & 3 \\
\hline 0 & 0 & 1 & 1 & 1 & 0 & 0 & 0 & 0 & 1 & 0 & 0 & & 4 & 0 \\
\hline
\end{tabular}

tructurar la cartera de servicios por zonas de planificación, "debido a que la cartera de servicios disponibles debe ser suficiente para responder a las necesidades de salud de la población” (Ministerio de Salud Pública 2012, 91). 


\section{Gráfico 2}

\section{Diagrama de árbol}

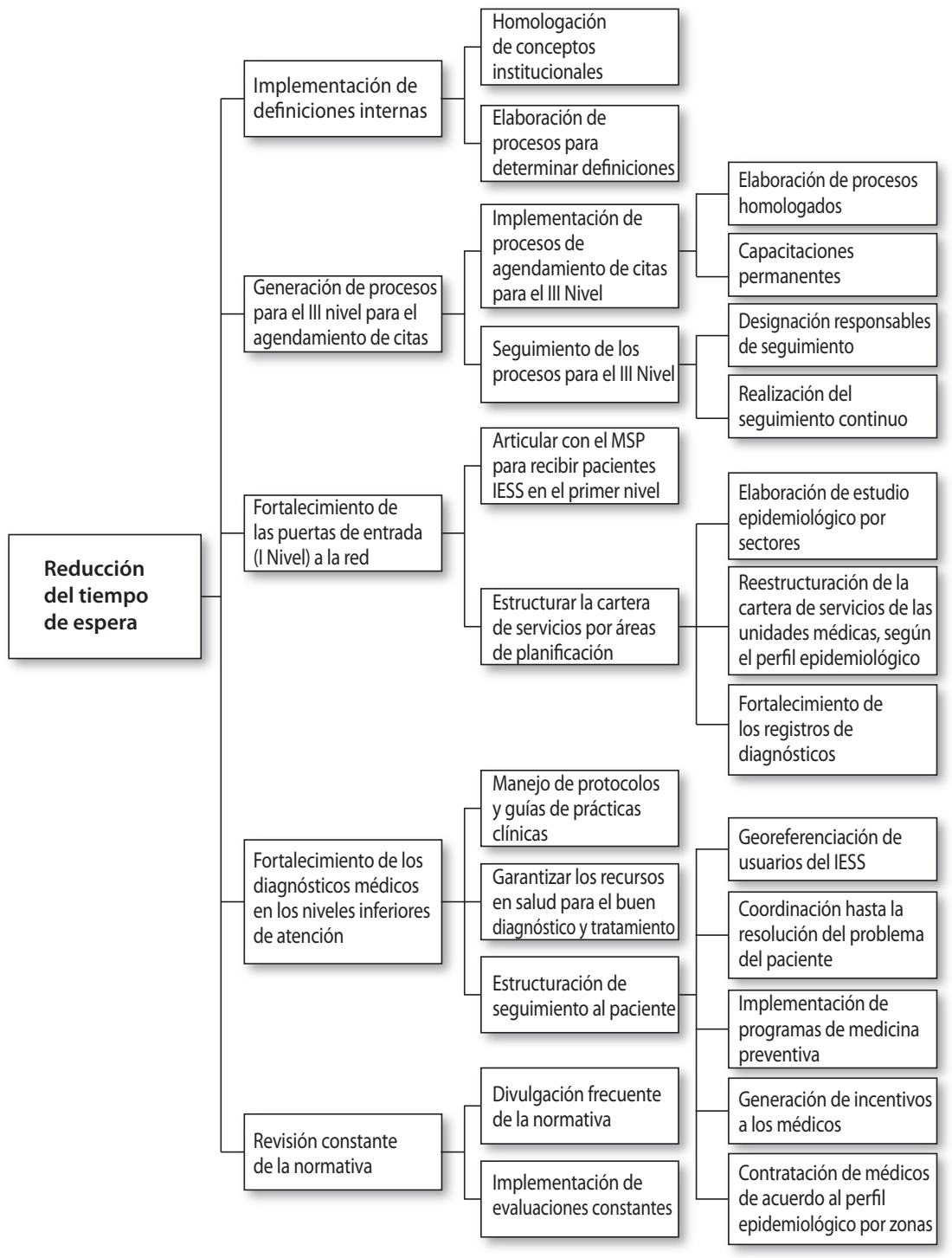

Elaboración propia. 
- Fortalecimiento de los diagnósticos médicos en los niveles inferiores de atención: es importante la implementación de protocolos y guías de prácticas clínicas, dentro de todas las unidades médicas, con la finalidad de obtener un diagnóstico y tratamiento oportuno, y resolver el problema en el mismo nivel.

Otro punto es la garantía de los recursos necesarios (medicamentos, insumos, personal, entre otros), evitando trámites engorrosos.

Otra acción es impulsar la salud preventiva y el monitoreo del paciente mediante redes e incentivos para los médicos del IESS, responsabilizándoles la salud de los usuarios asignados por territorio (evitando que se enfermen) y georreferenciándolos.

- Revisión constante de la normativa: se debe establecer un plan de divulgación y capacitación permanente. Así también, se propone que se realicen evaluaciones frecuentes para medir el conocimiento de los servidores con respecto a la normativa (ver gráfico 3).

\section{Diagrama de decisiones}

En el gráfico no se muestra la secuencia de pasos que se proponen para resolver el problema. Se han identificado nueve potenciales riesgos y sus contramedidas. Estas han sido categorizadas como: complicadas (x) o ejecutables (o). A continuación, el detalle:

- Generación de procesos sistematizados para la gestión de citas médicas, en las unidades del III nivel: el riesgo es la delegación de responsabilidad a funcionarios no capacitados. La contramedida, ejecutable, es la existencia de un proceso de selección de personal, mediante concurso de méritos, garantizando el mejor perfil.

- Fortalecimiento de las puertas de entrada (I Nivel) a la red de salud: la recomendación para este riesgo es la elaboración de auditorías médicas constantes, seleccionadas aleatoriamente por cada funcionario de salud y garantizar el buen registro del diagnóstico.

Finalmente, dentro de este grupo de recomendaciones, también se encuentra la reestructuración de la cartera de servicios en las unidades del IESS; esta es una actividad complicada de ejecutar porque depende de fac- 
Gráfico 3

Diagrama de decisiones

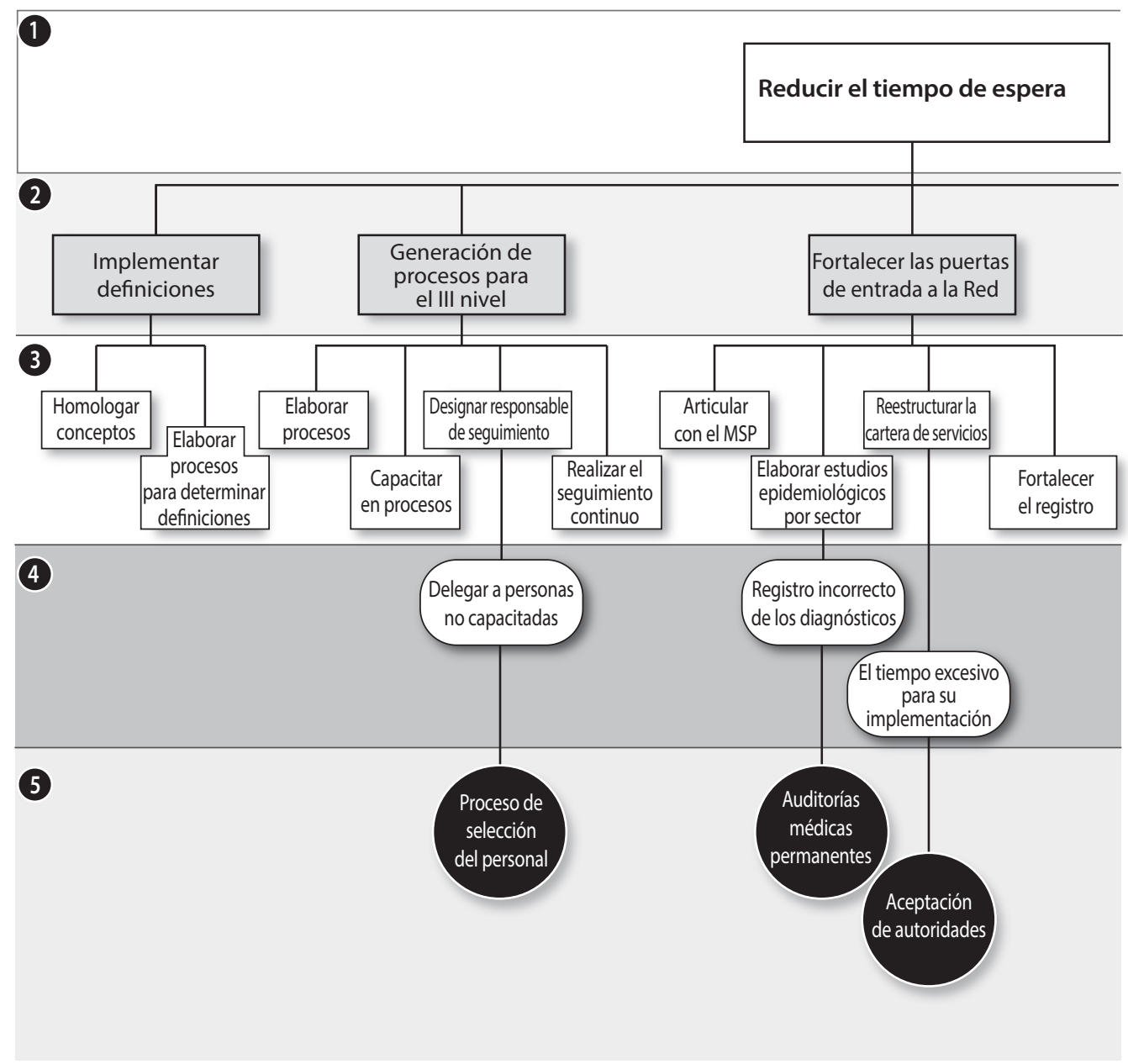

(1) Objetivo 2 Opciones de implementación

(4) Problemas que pueden aparecer
3 Secuencia de pasos de implementación

(5) Contramedidas

Elaboración propia. 
Un problema social: tiempos de espera en la consulta externa del Hospital Carlos Andrade Marín

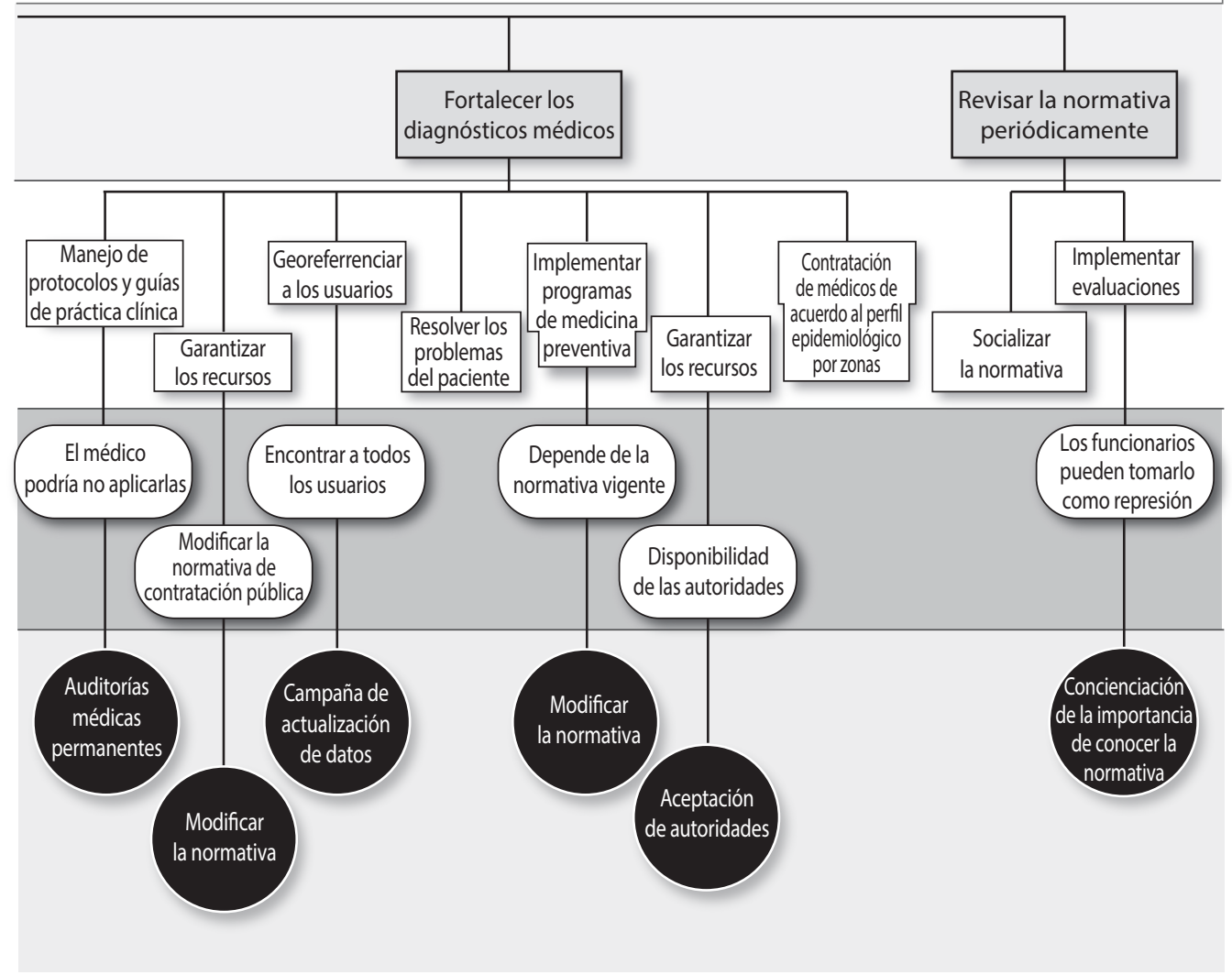


tores externos, e implica mover recursos hospitalarios. Además, depende de las autoridades de turno. Por tal razón, la contramedida es coordinar para disponer del apoyo de las autoridades, resultando ser complicado.

- Fortalecimiento de los diagnósticos médicos, de tal manera que sean acertados y oportunos: para disponer de los recursos necesarios, la contramedida es complicada porque se propone modificar la normativa, para facilitar las adquisiciones para el sector de la salud.

Otro posible riesgo es la falta de localización de los pacientes; se debe realizar una campaña comunicacional de actualización de datos. El registro debe ser fácil y accesible (internet, domiciliario, en islas de atención al cliente, etc.).

Al impulsar la medicina preventiva, y los incentivos médicos, depende de la decisión de las autoridades. Esto resulta ser complejo por lo que se recomienda la articulación y coordinación con las respectivas autoridades y modificaciones a la normativa.

- Revisión periódica de la normativa por los funcionarios relacionados con

el proceso: los funcionarios pueden ver la recomendación como impositiva; por tal razón, la contramedida recomendada es sensibilizar al personal, respecto a la importancia de aplicar la normativa legal (ver tabla 3).

La matriz de planeación y mejora continua propone la forma de ejecución de las actividades planteadas en los diagramas determinados previamente, de manera que detalla los actores y tiempos estimados de la puesta en marcha del plan de acción que se ha levantado para este documento.

\section{Conclusiones}

La salud es un derecho de las personas que se reconoce dentro de los convenios internacionales y dentro de la normativa del Ecuador. La provisión de la salud presenta desafíos ya que el sistema de salud nacional ha tenido limitaciones relacionadas con fragmentación y limitada articulación; para solucionar este panorama, desde el MAIS, se ha organizado la atención de la salud en cuatro niveles. 


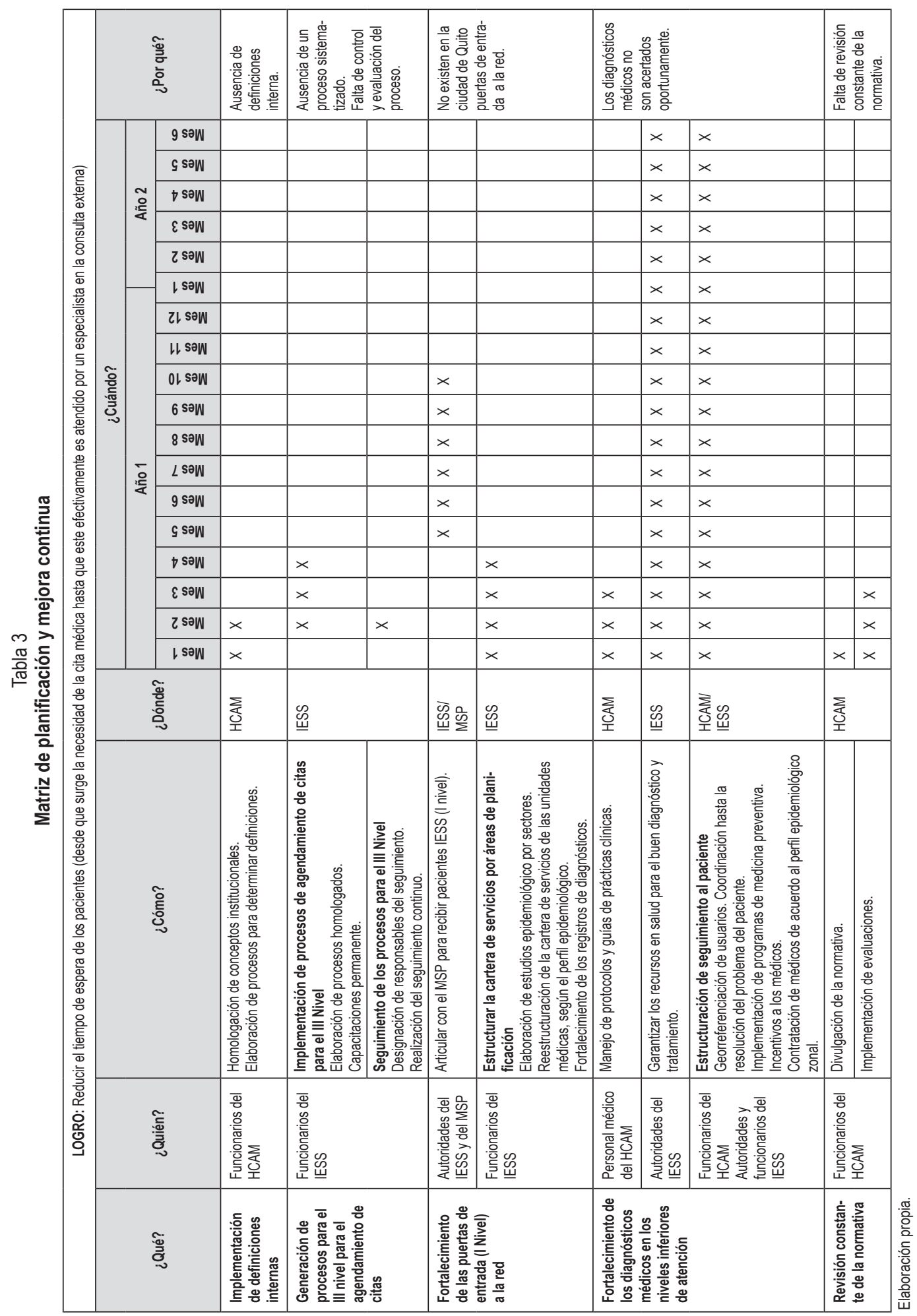


Se ha evidenciado que, para la asignación de turnos, han existido importantes problemas, desde la falta de monitoreo y seguimiento de los procesos involucrados, la débil articulación entre las unidades médicas, ya que existen casos de pacientes que no son referidos a tiempo, o en su defecto han recibido atención médica cuando su patología no corresponde al nivel de atención, hasta la falta de la capacidad instalada necesaria para atender a la demanda actual de las unidades médicas; estas causales no han podido ser superadas, de manera que se pueda fortalecer la gestión interna, para la resolución de la problemática.

Como parte de los hallazgos determinados, en relación con los problemas del tiempo de espera prolongado, es importante indicar que no son solo el resultado de las gestiones o decisiones ejecutadas por los administradores del hospital, sino también existen responsabilidades compartidas con actores externos, quienes están involucrados en el proceso, tales como autoridades nacionales del IESS y del MSP. De manera que los factores encontrados no solo tienen un impacto negativo en el hospital, sino también al resto de las unidades médicas, cuya principal causa es la débil gestión y seguimiento dentro de la articulación en la red.

Después de levantar varios elementos que han permitido determinar las principales causas de la problemática se tiene que existe ausencia de definiciones internas; ausencia de un proceso sistematizado para las unidades médicas del III nivel; falta de control y evaluación del proceso para la mejora continua; no existen en la ciudad de Quito puertas de entrada (nivel I) a la red para la atención en salud; los diagnósticos médicos no son acertados oportunamente; falta de revisión constante de la normativa por parte de los funcionarios. Estas acciones han involucrado a varios responsables y para lograr resultados se requiere de estrategias implementadas dentro y fuera de la casa de salud, las cuales no resultan ser fáciles de trabajarlas por incluir actores externos, además de que han sido debilidades que han estado presente por tiempos prolongados y han logrado institucionalizarse en el HCAM y demás unidades médicas.

Existen propuestas que han sido presentadas para poder solventar la problemática, de manera que cada una de estas ha pasado por una revisión para considerar su complejidad y estrategia de acción para su ejecución, las cuales están resumidas en cinco ítems, tales como la implementación de definicio- 
nes internas; generación de procesos homologados para el agendamiento de citas; fortalecimiento de puertas de entrada a la red de salud; mayor precisión de los diagnósticos médicos; y revisión constante de la normativa.

El estudio realizado ha permitido determinar causas y efectos que se han encontrado en el proceso de asignación de turnos de los pacientes. Ambos pueden ser reducidos mediante una secuencia de actividades que implican el trabajo conjunto entre las casas de salud, autoridades del IESS y la autoridad rectora. Mientras no se dé esta cohesión, no se podrá resolver el problema. La codependencia actual ha hecho que se dilaten los procesos o las propuestas presentadas, debido a las diferentes autorizaciones que se deben requerir para poder implementar alternativas de solución desde las unidades médicas del IESS; así mismo se ha evidenciado la falta de estrategias efectivas por parte de las entidades regidoras para resolver esta problemática en común. En ese sentido, solamente un trabajo articulado podrá reducir los tiempos de espera.

Esta propuesta resulta ser el punto de partida para futuras investigaciones, las cuales deben enfocarse a resolver integralmente la gestión de atención de los pacientes, de manera que el usuario tenga un diagnóstico y tratamiento, oportuno y de calidad. Adicionalmente, se deben buscar alternativas para la correcta articulación con las unidades médicas del primer y segundo nivel, las cuales tienen problemáticas similares y al funcionar como red cualquier problema que afecte en alguna de las instancias impactan en el resto de hospitales.

\section{Referencias}

Blandón, Laura. 2013. "Diseño e implementación de mecanismos interacción entre procesos de la unidad estratégica de negocio del gas natural de EMP: Antioquia”. Tesis de pregrado en Ingeniería Industrial. Corporación Universitaria Lasallista, Caldas, Antioquia.

Camisón, César, Sonia Cruz y Tomás González. 2006. Gestión de la calidad: conceptos, enfoques, modelos y sistemas. Madrid: Pearson Educación.

Comité Europeo de Normalización UE. 2015. Norma de sistema de gestión de calidad - ISO 9001. Norma de Sistema de Gestión de Calidad - ISO 9001. Madrid: Lloyd's Register Group.

EC. 2008. Constitución de la República del Ecuador. Registro Oficial 449, 20 de octubre. 
---. 2010. Ley de Seguridad Social. Registro Oficial 180, Suplemento, 10 de febrero.

Gutiérrez, Sandra, Diego Recalde, Ana Guevara y Gabriela Rivadeneira. 2009. “Optimización del sistema hospitalario ecuatoriano: estudio, modelización, simulación y minimización de tiempos de espera de pacientes de consulta externa". Revista Politécnica 30, No. 1: 83-90.

Hermida, Jorge, Carmen Laspina y Franklin Idrovo. 1996. "Reducción de los tiempos de espera mediante técnicas de garantía de la calidad en La Troncal, Ecuador". Bol Oficina Sanit Panam 121, No. 2: 95-101.

Ministerio de Salud Pública de Ecuador. 2012. Manual del modelo de atención integral de salud. Quito: Subsecretaría Nacional de Gobernanza de la Salud Pública del Ecuador.

Organización de las Naciones Unidad (ONU). 1948. Declaración Universal de los Derechos Humanos. Nueva York: ONU.

Pérez, José. 2004. Gestión por procesos: cómo utilizar la ISO 9001:2000 para mejorar la gestión de la organización. Madrid: ESIC Editorial.

Velázquez, Borja, y Viviana Vinueza. 2017. “Aplicación de modelos de teorías de colas a la gestión asistencial en los centros de salud". Enfermería Investiga 2, No. 1: 28-33.

Vilar, José, Fermín Gómez y Miguel Tejero. 1997. Las siete nuevas herramientas para la mejora de la calidad. Madrid: Fundación Confemetal. 